Draft version May 30, 2018

Preprint typeset using $\mathrm{IAT}_{\mathrm{E}} \mathrm{X}$ style emulateapj v. 12/14/05

\title{
THE SPLASH SURVEY: A SPECTROSCOPIC ANALYSIS OF THE METAL-POOR, LOW-LUMINOSITY M31 DSPH SATELLITE ANDROMEDA X ${ }^{1,2}$
}

\author{
Jason S. Kalirai ${ }^{3,4}$, Daniel B. Zucker ${ }^{5,6,7,8}$, Puragra Guhathakurta ${ }^{4}$, Marla Geha ${ }^{9}$, \\ Alexei Y. KniazeV ${ }^{5,10,11}$, David Martínez-Delgado ${ }^{5,12,13}$, Eric F. Bell ${ }^{5}$ \\ Eva K. Grebel ${ }^{14}$, and Karoline M. Gilbert ${ }^{15}$ \\ Draft version May 30, 2018
}

\begin{abstract}
Andromeda X (And X) is a newly discovered low-luminosity M31 dwarf spheroidal galaxy (dSph) found by Zucker et al. (2007) in the Sloan Digital Sky Survey (SDSS - York et al. 2000). In this paper, we present the first spectroscopic study of individual red giant branch stars in And X, as a part of the SPLASH Survey (Spectroscopic and Photometric Landscape of Andromeda's Stellar Halo). Using the Keck II telescope and multiobject DEIMOS spectrograph, we target two spectroscopic masks over the face of the galaxy and measure radial velocities for $\sim 100$ stars with a median accuracy of $\sigma_{v}$ $\sim 3 \mathrm{~km} \mathrm{~s}^{-1}$. The velocity histogram for this field confirms three populations of stars along the sight line: foreground Milky Way dwarfs at small negative velocities, M31 halo red giants over a broad range of velocities, and a very cold velocity "spike" consisting of 22 stars belonging to And X with $v_{\text {rad }}=-163.8 \pm 1.2 \mathrm{~km} \mathrm{~s}^{-1}$. By carefully considering both the random and systematic velocity errors of these stars (e.g., through duplicate star measurements), we derive an intrinsic velocity dispersion of just $\sigma_{v}=3.9 \pm 1.2 \mathrm{~km} \mathrm{~s}^{-1}$ for And X, which for its size, implies a minimum mass-to-light ratio of $M / L_{V}=37_{-19}^{+26}$ assuming the mass traces the light. Based on the clean sample of member stars, we measure the median metallicity of And $\mathrm{X}$ to be $[\mathrm{Fe} / \mathrm{H}]=-1.93 \pm 0.11$, with a slight radial metallicity gradient. The dispersion in metallicity is large, $\sigma\left([\mathrm{Fe} / \mathrm{H}]_{\mathrm{phot}}\right)=0.48$, possibly hinting that the galaxy retained much of its chemical enrichment products.
\end{abstract}

And X has a total integrated luminosity $\left(M_{V}=-8.1 \pm 0.5\right)$ that straddles the classical Local Group dSphs and the new SDSS ultra-low luminosity galaxies. The galaxy is among the most metal-poor dSphs known, especially relative to those with $M_{V}<-8$, and has the second lowest intrinsic velocity dispersion of the entire sample. Our results suggest that And $\mathrm{X}$ is less massive by a factor of four when compared to Milky Way dSphs of comparable luminosity (e.g., Draco and Ursa Minor). We discuss the potential for better understanding the formation and evolution mechanisms for M31's system of dSphs through (current) kinematic and chemical abundance studies, especially in relation to the Milky Way sample.

Subject headings: dark matter - galaxies: abundances - galaxies: dwarf-galaxies: individual (And X) - techniques: spectroscopic

\begin{abstract}
${ }^{1}$ Data presented herein were obtained at the W. M. Keck Observatory, which is operated as a scientific partnership among the California Institute of Technology, the University of California, and the National Aeronautics and Space Administration. The Observatory was made possible by the generous financial support of the W. M. Keck Foundation.

2 Based on observations made with the Nordic Optical Telescope, operated on the island of La Palma jointly by Denmark, Finland, Iceland, Norway, and Sweden, in the Spanish Observatorio del Roque de los Muchachos of the Instituto de Astrofísica de Canarias; these observations were funded by the Optical Infrared Coordination Network (OPTICON), a major international collaboration supported by the Research Infrastructures Programme of the European Commission's Sixth Framework Programme.

${ }^{3}$ Space Telescope Science Institute, 3700 San Martin Drive, Baltimore MD, 21218; jkalirai@stsci.edu

${ }^{4}$ University of California Observatories/Lick Observatory, University of California at Santa Cruz, 1156 High Street, Santa Cruz CA, 95064, USA; raja@ucolick.org

${ }^{5}$ Max-Planck-Institut für Astronomie, Königstuhl 17, D-69117 Heidelberg, Germany; bell@mpia.de

${ }^{6}$ Institute of Astronomy, University of Cambridge, Madingley Road, Cambridge, CB3 0HA, United Kingdom

7 Department of Physics, Macquarie University, North Ryde, NSW 2109, Australia; zucker@science.mq.edu.au

8 Anglo-Australian Observatory, PO Box 296, Epping, NSW 1710, Australia

${ }^{9}$ Astronomy Department, Yale University, New Haven CT,
\end{abstract}

\section{INTRODUCTION}

A census of dwarf galaxies in the neighborhoods of large systems such as the Milky Way and Andromeda (M31) can yield important constraints on the processes that shaped massive galaxies. Current predictions from $\Lambda \mathrm{CDM}$ simulations suggest that at least an order of magnitude more satellites should be present around these systems than has been observed (e.g., Klypin et al. 1999; Moore et al. 1999). Several explanations for this "missing satellite problem" have been suggested, both on the

\footnotetext{
06510; marla.geha@yale.edu

10 South African Astronomical Observatory, PO Box 9, 7935 Observatory, Cape Town, South Africa; akniazev@saao.ac.za

${ }^{11}$ South African Large Telescope Foundation, PO Box 9, 7935 Observatory, Cape Town, South Africa

12 Instituto de Astrofísica de Canarias, La Laguna, Spain; ddelgado@iac.es

${ }^{13}$ Ramón y Cajal Fellow

14 Astronomisches Rechen-Institut, Zentrum für Astronomie der Universität Heidelberg, Mönchhofstr. 12-14, D-69120 Heidelberg, Germany; grebel@ari.uni-heidelberg.de

${ }^{15}$ Department of Astronomy, Box 351580, University of Washingon, Seattle WA, 98195; kmgilber@u.washington.edu
} 
theoretical and observational fronts. For example, many of the predicted subhalos may be entirely "dark", and therefore may not have experienced any star formation (e.g., Somerville 2002), and/or our census of these lowluminosity galaxies may be highly incomplete.

Recent wide field surveys in both the Milky Way and M31 have confirmed the latter possibility. In our Galaxy, the number of dwarf spheroidal galaxies (dSphs) known has doubled in the past few years, thanks in large part to the Sloan Digital Sky Survey (SDSS - York et al. 2000; e.g., Willman et al. 2005; Zucker et al. 2006a, Belokurov et al. 2006; Zucker et al. 2006b; Belokurov et al. 2007; Walsh, Jerjen, \& Willman 2007). Similarly, the last three years has seen the number of dSphs in M31 grow by more than a factor of two (Zucker et al. 2004b, 2007; Martin et al. 2006; Majewski et al. 2007; Ibata et al. 2007; McConnachie et al. 2008). Yet, considering the limited regions of the Milky Way and M31 that have been surveyed for these lower luminosity galaxies, many more such galaxies should be discovered in the coming years (e.g., with Pan-STARRS, the Southern Hemisphere Stromlo Missing Satellites Survey on the SkyMapper Telescope - Keller et al. 2007, and the Large Synoptic Survey Telescope, see Tollerud et al. 2008 for expected number counts).

None of the newly discovered Milky Way dSphs in the SDSS are directly seen in the initial images, but rather they are detected as resolved stellar overdensities. Deeper follow up images have revealed that some of these galaxies are distinct in their properties as compared to the existing sample; they are more irregular and have very low surface brightnesses $\left(\mu_{V}>27 \mathrm{mag} \operatorname{arcsec}^{-2}\right)$. The faintest objects, such as Willman 1 and Segue 1, have an integrated luminosity less than a single bright red giant, and in an $M_{V}$ vs half-light radius plane, appear to bridge globular clusters and dwarf galaxies (Belokurov et al. 2007). Several investigations are now underway to understand the detailed nature of these systems (e.g., mean abundance, abundance spread, velocities, and mass-to-light ratios) through follow up spectroscopic observations (e.g., Simon \& Geha 2007; Koch et al. 2008a; Norris et al. 2008; Geha et al. 2009).

In distinction to the Milky Way satellites, there have been limited spectroscopic observations of individual stars in M31 dSphs, with most studies only confirming a handful of members (Cote et al. 1999; Guhathakurta, Reitzel, \& Grebel 2000; Chapman et al. 2005; Majewski et al. 2007). Similar studies of M31 field stars in the SPLASH Survey have recently revealed that the metalrich inner spheroid of M31 dominates the metal-poor outer halo out to a much larger radius $(20-30 \mathrm{kpc})$ than in the Milky Way (Guhathakurta et al. 2005; Kalirai et al. 2006a). This disparity likely reflects a more violent accretion history in M31 as compared to our Galaxy (Hammer et al. 2007), the roots of which may lie in the properties of the accreted (and possibly unaccreted) satellite systems of the two galaxies. In fact, McConnachie \& Irwin (2006) show that, at a fixed luminosity, the scale radii of the M31 dSph galaxies is a factor of two larger than the canonical Milky Way satellites. If the dark matter masses of these galaxies are the same and the mass traces the light, this difference should be reflected in the velocity dispersions of the galaxies with the M31 sample being colder. However, alternate as-

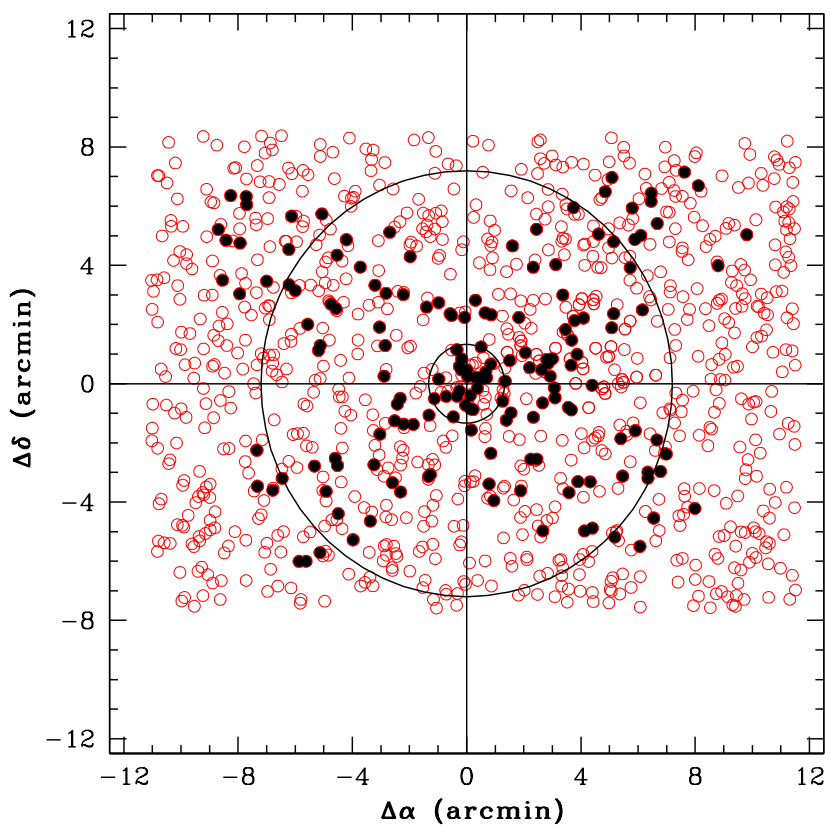

FIG. 1. - The selection of Keck/DEIMOS spectroscopic targets (filled circles) from the photometric catalog (open circles) of And X. The straight lines intersect at the center of And X $\left(\alpha_{\mathrm{J} 2000}\right.$ $\left.=01^{\mathrm{h}} 06^{\mathrm{m}} 39.20^{\mathrm{s}}, \delta_{\mathrm{J} 2000}=44^{\circ} 48^{\prime} 44.6^{\prime \prime}\right)$ and the two circles denote the core radius $(1 ! 33)$ and tidal radius $(7 ! 2)$ of the galaxy (Zucker et al. 2007). This "cross" pattern reflects the \pm 45 degree position angle of the two spectroscopic masks. This arrangement ensures the targeting of many stars near the center of And X as well as along spokes that extend to beyond the tidal radius of the galaxy.

sumptions on the spatial distribution of stars relative to the dark matter can lead to different conclusions, as discussed in $\S 5$.

To address some of these questions, our team has now begun a systematic survey of M31 dSphs with the Keck II telescope and DEIMOS multi-object spectrograph. At the distance of M31, the apparent angular size of a typical dSph is well suited for this instrument as we can easily target several hundred potential red giants simultaneously. To date, six of the M31 dSphs have been targeted, including And XIV which has been presented in Majewski et al. (2007) and And I, II, and III which will be presented in Kalirai et al. (2009). In this paper, we discuss the first spectroscopic study of individual red giants in the newly discovered, low-luminosity M31 dSph, And X (Zucker et al. 2007). Our target selection, observational design, and data reduction are presented in the next section, followed by an analysis of the And X velocity histogram to establish both the membership of individual stars and the mean velocity of the galaxy $(\S 3)$. We resolve the intrinsic central velocity dispersion of And X in $\S 3.3$ and calculate the mass-to-light ratio of the galaxy. Chemical abundances of the member stars in And X are calculated both photometrically and spectroscopically in $\S 4$. The results are discussed and summarized in $\S 5$.

\section{THE DATA}

\subsection{Observations}

And X was first discovered as a stellar overdensity on an SDSS imaging scan of the major axis of M31 (extending $18^{\circ} \times 2.5^{\circ}$ ), observed on 5 October 2002 (Zucker et al. 2004a). The overdensity was only visible after 

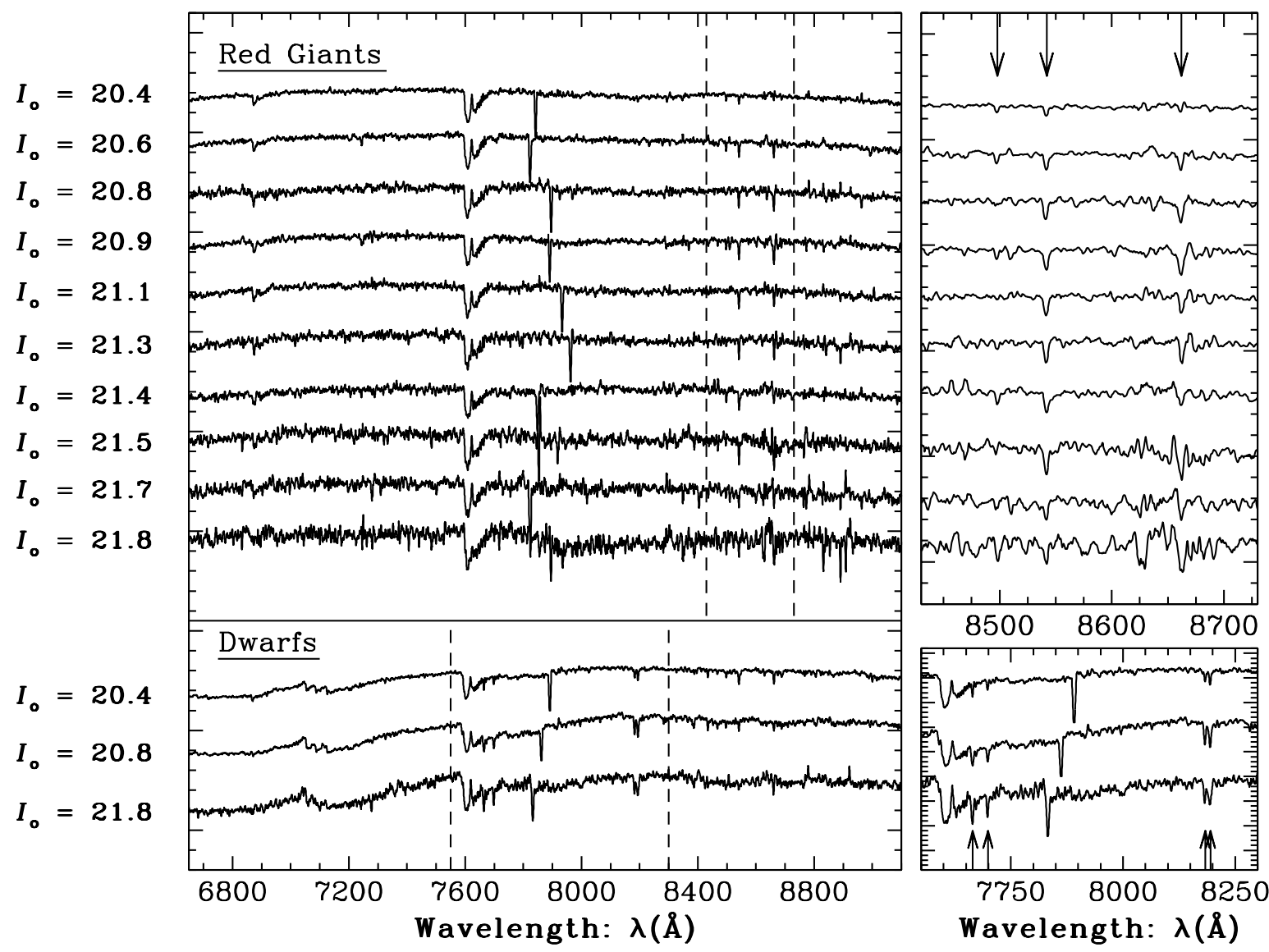

FIG. 2.- The spectra of red giants (top) and three foreground Milky Way dwarfs (bottom) in our And X data set, smoothed by a 10 pixel boxcar function and redshifted to zero velocity. The B-band and A-band atmospheric absorption features can be seen in the spectra at $6900 \AA$ and $7600 \AA$. A dip close to the center of each spectrum is caused by the CCD gap. The stars presented here have been chosen to reflect the full magnitude range over which we measure radial velocities. A closer look at some of the key spectral features for these stars is presented in the two panels on the right. For the giants (top), we display the region of the Ca II triplet, with an arrow at the top indicating the position of each line. For the dwarfs (bottom), we focus on the pressure sensitive Na I doublet (8190 A) and K I lines (7665 and $7699 \AA$ ) that are used to eliminate foreground contamination (see $\S 3.2$ ).

selecting objects with magnitudes and colors consistent with the red giant branch in a metal-poor population, at the distance of M31. Spatially, the overdensity lies along the projection of the N-E major axis at a distance of $\sim 80 \mathrm{kpc}$ from M31's center. These initial observations were followed up with subsequent imaging from the William Herschel Telescope and the Nordic Optical Telescope in late 2004. The resulting images confirmed the presence of a low surface brightness, spatially coherent group of stars with a color-magnitude diagram (CMD) indicating a blue red giant branch (i.e., presumably metal-poor stars). The integrated $V$-band luminosity of the galaxy is $M_{V}=-8.1 \pm 0.5$. Further details on these photometric observations are presented in Zucker et al. (2007), although we note here that our default photometric catalogue is taken to be the William Herschel Telescope data as the calibration is superior to the Nordic Optical Telescope data.

We used these photometric data as input for our multiobject spectroscopic observations with Keck II. The image of And X from the Nordic Optical Telescope shows the galaxy to have an extent of a few arcminutes. The estimated tidal radius by Zucker et al. (2007) is $\sim 7$ arcminutes, much smaller than the long-axis of the DEIMOS spectrograph, which extends $\sim 16$ arcminutes (see Figure 1). Therefore, we could easily observe the full extent of the galaxy with a single pointing. We selected targets based on their location in the CMD. The tip of the red giant branch in And $\mathrm{X}$ is located at $I_{0}=20.25(d=$ $701 \mathrm{kpc}$ ), and so our highest priority objects are those with $20.0<I_{0}<22.0$. Lower priorities are given to fainter stars.

Unfortunately, the stellar density over the And X field of view is not high enough to utilize the full capabilities of DEIMOS, an instrument that could easily target $\gtrsim 200$ stars in a single pointing. Although we can increase the number of And $\mathrm{X}$ targets by pushing to fainter limits along the red giant branch, our experience has shown that it is difficult to measure accurate radial velocities from such lower signal-to-noise data unless the integration times are made much longer. We therefore, generate a second spectroscopic mask at roughly the same position, but offset in position angle by 90 degrees (shown in Figure 1). This offers us two advantages. First, we can target mostly different stars in the two pointings to increase the number of reliable measurements near the tip of the red giant branch in And X. Second, we can obtain duplicate spectra for a few stars in order to constrain the 
TABLE 1

\begin{tabular}{|c|c|c|c|c|c|c|c|c|}
\hline$\overline{\overline{\text { Date }}}$ & "Mask & $\alpha_{J 2000}$ & $\overline{\overline{\delta_{J 2000}}}$ & Pos. Angle $\left({ }^{\circ}\right)$ & Exp. Time (s) & $\overline{\text { Seeing }}$ & Airmass & No. of Targets* \\
\hline 5 Sept 2005 & d10_1 & $01: 06: 39.20$ & $44: 48: 44.6$ & 45 & $3 \times 1200$ & $0 . \prime 65-0 . ! 77$ & $1.26-1.41$ & 85 \\
\hline 5 Sept 2005 & d10_2 & 01:06:39.20 & $44: 48: 44.6$ & -45 & $3 \times 1200$ & $0 .{ }^{\prime \prime} 51-0 . \prime 60$ & $1.14-1.21$ & 94 \\
\hline
\end{tabular}

* 14 objects were targeted on both masks.

uncertainties in our radial velocity measurements based on independent measurements. In total, we targeted 85 objects on the first mask (d10_1) and 94 objects on the second mask (d10_2), 14 of which were placed on both masks.

We observed each of these two spectroscopic masks for 1 hour on 5 September 2005 (see Table 1). The individual exposure times were set at 1,200 seconds. The observing conditions were photometric, the airmass was low at 1.2 - 1.4, and the seeing was exceptional at 0. . $51-0$. ". 65 for most of the observations, and at worst $0 . \prime 77$ for one exposure. The observations were obtained using our standard setup, as described in several previous papers (e.g., Gilbert et al. 2006). This utilizes the 1200 lines $\mathrm{mm}^{-1}$ grating at a central wavelength of $7800 \AA$, with a wavelength range of approximately $\approx 6400-9100 \AA$ for each star.

\subsection{Data Reduction}

The two DEIMOS multi-slit masks for And X were reduced using the spec2d software pipeline developed by the DEEP2 team at the University of California-Berkeley (see Faber et al. 2007 for details). To summarize the key steps, the flat-field exposures are first used to rectify the curved raw spectra into rectangular arrays by applying small shifts and interpolating in the spatial direction. A one-dimensional slit function correction and two-dimensional flat-field and fringing correction are applied to each slitlet. Using the DEIMOS optical model as a starting point, a two-dimensional wavelength solution is determined from the arc lamp exposures with residuals of order $0.01 \AA$. Each slitlet is then sky-subtracted exposure by exposure using a B-spline model for the sky. The individual exposures of the slitlet are averaged with cosmic-ray rejection and inverse-variance weighting. Finally one dimensional spectra are extracted for all science targets using the optimal scheme of Horne (1986) and are rebinned into logarithmic wavelength bins with $14 \mathrm{~km} \mathrm{~s}^{-1}$ per pixel. Example spectra for several stars are shown in Figure 2.

We measure radial velocities for all objects by crosscorrelating the spectra with high signal-to-noise stellar templates. The methods used for this analysis are described in detail in Simon \& Geha (2007). Specifically, we note that a correction is made to offset any wavelength calibration error that results from imprecise astrometry. This is performed by cross-correlating each spectrum with several bright, hot standard stars. These stars exhibit a nearly featureless spectrum, but with a clean detection of the telluric lines in the A-band absorption feature. The resulting cross-correlation leads to an estimate of how much the object was offset from the center of the slit and therefore provides a means to correct any resulting wavelength (and therefore velocity) error (see also Sohn et al. 2006 for further details).

Of the 179 spectra obtained, this cross-correlation yielded an accurate velocity for just over half of the stars (98 in total). We manually verified each of the template fits and only kept those which showed at least two definite spectral features in common. In fact, for most objects multiple absorption features were matched over the $3000 \AA$ wavelength range. Additionally, we kept 15 objects in the group of 98 whose spectra showed at least one definite match to the template and a second marginal match (see discussion in Guhathakurta et al. 2006; Kalirai et al. 2006b). The objects for which this failed typically have poor signal-to-noise spectra and are predominantly the fainter targets $\left(I_{0}>22\right)$ in our study. Finally, we applied a heliocentric correction to each star's velocity.

Of the original 14 duplicate stars, 7 have measured velocities on both masks, one of which is in the marginal category discussed above. The standard deviation in the repeat velocity measurements between the two independent measurements for these objects is $4.1 \mathrm{~km} \mathrm{~s}^{-1}$, and the mean error in velocity among the 14 measurements is $4.0 \mathrm{~km} \mathrm{~s}^{-1}$. The differences are not systematically correlated with any one set of observations. For our final velocity catalog, we calculate the weighted mean velocity of each of the duplicate pairs and therefore have 91 individual stars.

\section{KINEMATICS}

\subsection{Radial Velocity Accuracy}

Without careful treatment, radial velocities derived from relatively low resolution spectra can have individual velocity uncertainties of at least several $\mathrm{km} \mathrm{s}^{-1}$. In fact, not applying the A-band absorption correction discussed earlier would alone translate to an uncertainty of $\sim 3 \mathrm{~km} \mathrm{~s}^{-1}$ for these data. This has been directly tested by Simon \& Geha (2007) by comparing the measured velocities of duplicate stars before and after the correction. As typical low-luminosity Milky Way dSphs are kinematically cold, with velocity dispersions of $\sigma_{v} \lesssim 10 \mathrm{~km} \mathrm{~s}^{-1}$, an accurate measurement of the radial velocities, and an understanding of the velocity errors, are crucial to probe the internal kinematics of these galaxies.

We measure the errors in our velocity measurements using the method discussed by Simon \& Geha (2007). First, we generate 500 spectra with artificial noise at each pixel scaled by the observed variance in that pixel, for each star. This Monte Carlo leads to an error bar which is defined as the square root of the variance recovered from re-fitting these 500 spectra. The error estimates are next compared to the difference in velocity of the duplicate measurements to establish the contribution from any additional error sources that are not accounted for in the Monte Carlo method. Simon \& Geha (2007) estimated this "floor" to be $\epsilon=2.2 \mathrm{~km} \mathrm{~s}^{-1}$ in their data set. This implies that the observed difference in velocity measured for duplicate stars (taken to be the true error in the measurements), is reproduced by adding the 

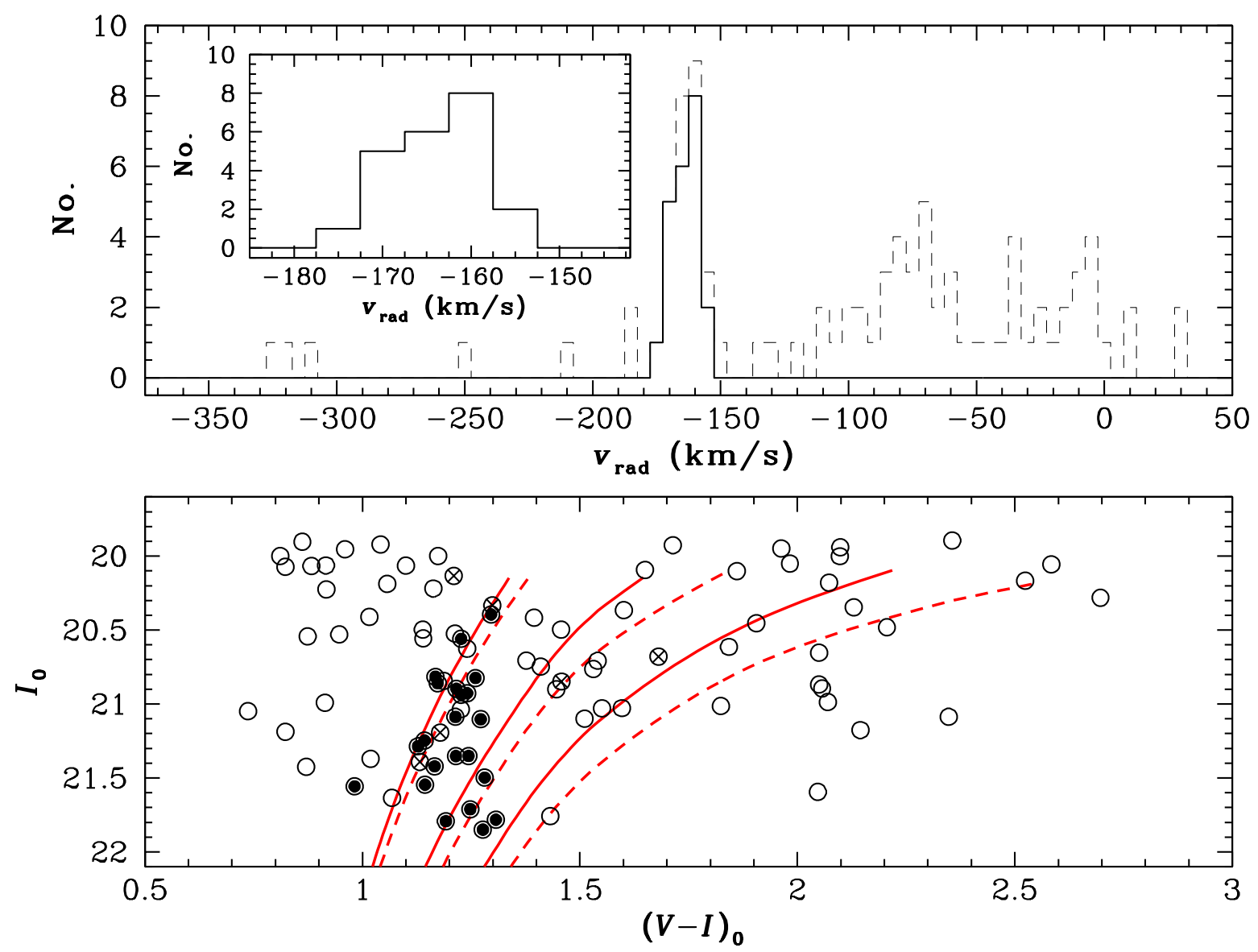

FIG. 3. - Top - The velocity histogram of all objects measured along the line of sight (dashed line) clearly indicates a kinematically cold population of stars with $v_{\mathrm{rad}}=-163.8 \pm 1.2 \mathrm{~km} \mathrm{~s}^{-1}$, and $\sigma_{v}=3.9 \pm 1.2 \mathrm{~km} \mathrm{~s}^{-1}$. This velocity "spike" represents And X. At smaller negative velocities, a large number of Milky Way dwarfs are present in the data set. These objects, as well as candidate M31 halo giants at larger negative velocities, are removed as discussed in $\S 3.2$ to yield the final And X velocity histogram (solid line). A finer scale, centered on the dSph's radial velocity, is shown in the inset. Bottom - And X velocity members (filled circles) are found to lie in a tight sequence consistent with a metal-poor red giant branch population. The solid red curves indicate $[\alpha / \mathrm{Fe}]=0.0$ isochrones (dashed are for $[\alpha / \mathrm{Fe}]$ $=+0.3$ ) with metallicities of $[\mathrm{Fe} / \mathrm{H}]=-2.31$ (left), -1.31 (middle), and -0.83 (right) and ages of 12 Gyr from Vandenberg, Bergbusch, \& Dowler (2006). The open circles designate the non-member stars along the line of sight and the six crosses with open circles mark the objects that we further rejected from the initial sample as discussed in $\S 3.2$. Stars with $I_{0} \gtrsim 21.5$ are affected by larger photometric errors, $\sigma_{(V-I)} \sim 0.2$, as discussed in the text.

quadrature sum of the Monte Carlo error estimates with a $2.2 \mathrm{~km} \mathrm{~s}^{-1}$ additional error bar. For our duplicates (including stars observed in And I, II, and III; will be presented Kalirai et al. 2009), we measure a value of $\epsilon \lesssim$ $3 \mathrm{~km} \mathrm{~s}^{-1}$, very similar to Simon \& Geha (2007). As their measurement is based on a factor of three larger sample than ours, we fold in a $2.2 \mathrm{~km} \mathrm{~s}^{-1}$ error to the measured Monte Carlo values.

As discussed in $\S 2.3$ of Simon \& Geha (2007), a comparison of the velocities and velocity dispersions of kinematically cold populations with measurements and errors as described above yields excellent agreement with independent high-resolution analysis. Specifically, for a similar number of stars, the measured velocity dispersion of the globular cluster NGC 2419 using Keck/DEIMOS in this setup is identical to HIRES measurements discussed in Simon \& Geha (2007). Similarly, the comparison of a half dozen Keck/DEIMOS red giant spectroscopic measurements to high-resolution analysis of the same stars in UMa I by Kleyna et al. (2005) indicates differences that are smaller than the $1 \sigma$ uncertainty. These results illustrate that the method above can be used to recover velocity dispersions as low as $2.3 \mathrm{~km} \mathrm{~s}^{-1}$.

\subsection{Membership and the Mean Velocity of And X}

Zucker et al. (2007) estimate the distance modulus of And $\mathrm{X}$ to be $(m-M)_{0} \sim 24.23$ from the tip of the red giant branch. This places And $\mathrm{X} \sim 80 \mathrm{kpc}$ in front of M31. Our line of sight towards And $\mathrm{X}$ will therefore first intersect the disk and halo of the Milky Way, then And X, and finally M31's stellar halo. The method that we have used to select targets is based on the photometric properties of the stars, and therefore our resulting radial velocity catalog contains a mix of stars belonging to each of these three stellar populations (e.g., a nearby Milky Way dwarf can mimic an M31 red giant in the CMD). Fortunately, the radial velocity signatures of each of these groups of stars is expected to be different.

The radial velocity histogram for the 91 stars in our final catalog is presented as a dashed line in Figure 3 (top). The most salient feature in the diagram is the kinematically cold "spike" in the center. This velocity peak represents And X and therefore kinematically confirms the presence of a new dwarf satellite galaxy in the 
Zucker et al. (2007) imaging study. In addition to this population, we see an asymmetric velocity distribution around And X. Many more stars are found at smaller negative velocities, suggesting that a significant number of Milky Way dwarfs are in our sample. This is expected for several reasons. First, And X is a low luminosity galaxy and only occupies a small fraction of each of our DEIMOS masks, the rest of which is filled predominantly with non-member stars (see Figure 1). Second, the input photometry that we used was not pre-screened to eliminate possible dwarfs based on their DDO51 photometry, as was done by members of our group in studies of And XIV (Majewski et al. 2007) and M31's stellar halo (Guhathakurta et al. 2005; Kalirai et al. 2006a).

Gilbert et al. (2006) show that the bulk of the Milky Way dwarfs along M31's line of sight have a small negative radial velocity. Still, these stars can overlap the much (dynamically) hotter spheroid or halo of M31, which has a mean velocity of $v_{\text {rad }}=-300 \mathrm{~km} \mathrm{~s}^{-1}$. Gilbert et al. (2006) go on to develop a method based on several photometric and spectroscopic diagnostics that efficiently cleans out the foreground dwarf star contamination from M31 halo giants. Specifically, this method combines information from the velocity histogram, colormagnitude diagram, metallicity measurements using two approaches, and also incorporates the strengths of several spectral features to yield a clean sample of giants. For example, the measurement of the Na I doublet at $8190 \AA$ and the KI absorption lines at 7665 and $7699 \AA$ help yield excellent discrimination between cool dwarfs and giants as these lines are pressure sensitive, and therefore strong in dwarfs and absent in giants. These absorption lines can be easily seen in the raw spectra of the cooler dwarfs, even with the overlapping telluric absorption. For this sight line, we use an analogous method to Gilbert et al. (2006) to weed out stars that are Milky Way dwarfs and therefore, not associated with And X. The major difference in our case is that we are not targeting a kinematically diffuse M31 halo population, but rather a cold spike representing And X. This difference is applied to the Gilbert et al. (2006) formalism by adjusting the "training set" to represent a Gaussian with $\sigma_{v}$ $=20 \mathrm{~km} \mathrm{~s}^{-1}$, centered on the rough velocity of And X (we used $-170 \mathrm{~km} \mathrm{~s}^{-1}$ for this). The resulting classification of a member star vs non-member dwarf is not sensitively dependent on the choice of these parameters, as long as the initial guesses are not too restrictive. In this refined method, we have also adjusted the distance of the population to And X's known value (e.g., in computing photometric metallicities, see $\S 4$ ).

With this initial classification, we find 28 stars that can be considered giants in this data set. However, a small number of these stars are expected to be M31 field halo members, unassociated with And X. To ensure as secure a sample as possible, even at the expense of possibly losing one or two member stars, we further eliminate four stars from this group of 28 . These stars all have velocities that are inconsistent at $>3 \sigma$ with the mean of the remaining And X sample, and three of them are also located more than three core-radii (and half the tidal radius) from the center of the dSph. These stars, likely M31 field halo stars or possibly some residual Milky Way dwarf contaminants, have velocities of $v_{\text {rad }}=-108.7 \mathrm{~km} \mathrm{~s}^{-1}$, $-122.4 \mathrm{~km} \mathrm{~s}^{-1},-185.5 \mathrm{~km} \mathrm{~s}^{-1}$, and $-209.5 \mathrm{~km} \mathrm{~s}^{-1}$. As a final cut, we eliminate two more objects with radial velocities that fall within the range of And $\mathrm{X}$ member stars, $v_{\text {rad }}=-158.6 \mathrm{~km} \mathrm{~s}^{-1}$ and $v_{\text {rad }}=-163.2 \mathrm{~km} \mathrm{~s}^{-1}$. The first object is also located more than three core-radii from the center of And X, and is both brighter and bluer than the expected tip of the red giant branch on the CMD (see next section). The second object is much redder than the red giant branch of And X and is the only remaining star showing evidence for both $\mathrm{NaI}$ and $\mathrm{K}$ I absorption features. As we show below, And X's radial velocity is almost exactly equal to the radial velocity of this star, and therefore our likelihood measurement was artificially biased into classifying this object as a member star. We stress that the mean velocity and velocity dispersion measured for And X are essentially unchanged if these two stars are left into the final sample, differing by $<0.4 \mathrm{~km} \mathrm{~s}^{-1}$ and $<0.2 \mathrm{~km} \mathrm{~s}^{-1}$ respectively (much smaller than the error bars, see below).

The final velocity histogram of the 22 member stars of And $\mathrm{X}$ is shown as a solid line in Figure 3 (top). The same plot over a finer velocity range and centered on the galaxy's mean velocity, where the small ticks are each $2.5 \mathrm{~km} \mathrm{~s}^{-1}$, is shown in the inset. Based on this secure sample of stars, the weighted mean radial velocity of And $\mathrm{X}$ is $v_{\mathrm{rad}}=-163.8 \pm 1.2 \mathrm{~km} \mathrm{~s}^{-1}$.

\subsubsection{Color-Magnitude Diagram}

The CMD for the stars along our And $\mathrm{X}$ sight line is presented in Figure 3 (bottom). This includes only those stars for which we measured a reliable velocity. The open circles mark those objects that we determined to be nonmembers of And X, most of which are Milky Way dwarfs. As expected, these are found over a broad color range in the CMD. The filled circles designate the confirmed And X members and the open circles with crosses signify the six stars that were further rejected from membership as discussed above.

The filled circles in Figure 3 (bottom) are distributed in a narrow sequence of the CMD, signifying the red giant branch of And X. To demonstrate this, we overlay three solid (dashed) curves on the CMD representing theoretical isochrones near the red giant tip with an age of 12 Gyr and $[\alpha / \mathrm{Fe}]=0.0([\alpha / \mathrm{Fe}]=+0.3)$ (Vandenberg, Bergbusch, \& Dowler 2006). The metallicities of these models are $[\mathrm{Fe} / \mathrm{H}]=-2.31$ (left), $[\mathrm{Fe} / \mathrm{H}]=-1.31$ (mid$\mathrm{dle}$ ), and $[\mathrm{Fe} / \mathrm{H}]=-0.83$ (right). For this comparison, we have shifted the isochrones to a distance modulus of $(m-M)_{0} \sim 24.23$ and reddened them by $E(V-I)=$ 0.21 (Zucker et al. 2007). Clearly, the And X stars are in good agreement with these metal-poor isochrones with the exception of the faintest stars with $I_{0} \gtrsim 21.5$. Four of these faintest objects fall significantly redder than the dominant sequence, and one star falls bluer than the most metal-poor isochrone by $\sim 0.1$ magnitudes.

The default photometry for 20 of our final member stars is derived from the William Herschel Telescope observations, whereas the remaining two stars are drawn from the Nordic Optical Telescope, both of which have $I_{0}>21.5$. In both catalogs, the CMD of all stars that were measured on the imaging frames indicates a clear increase in the spread of the red giant branch at $I_{0} \sim 21.5$, and so we estimate that the errors on the colors of these faintest stars are at least $\sigma_{(V-I)} \sim 0.2$ magnitudes. If we only consider the Nordic Optical Telescope data, we also 
find that two of the four faintest stars fall redder than an extension of the obvious red giant branch. We consider more carefully the use of these stars in our metallicity calculations in $\S 4$. For completeness, we point out that these fainter stars are members of And X as defined above and therefore the measured mean velocity (and intrinsic velocity dispersion) should minimally vary if they are removed from the sample. To test this, we confirmed shifts in the inferred mean velocity of $<0.7 \mathrm{~km} \mathrm{~s}^{-1}$ and in the calculated velocity dispersion (see below) of $<0.2 \mathrm{~km} \mathrm{~s}^{-1}$ if the sample is restricted to just the tight sequence of points that are blueward of the $[\mathrm{Fe} / \mathrm{H}]=-1.31$ isochrone (also excluding the very blue star at $\left.(V-I)_{0}=0.98\right)$. The characteristics of stars within several diagnostics including their radial distance from the center of And X, line of sight velocities, and location in the CMD are compared to one another in Figure 4 to illustrate the robustness of the membership criteria.

There are a few non-And $\mathrm{X}$ member stars in our overall sample with heliocentric velocities of -300 to $-350 \mathrm{~km} \mathrm{~s}^{-1}$. These objects are unlikely to be Milky Way dwarfs based on their kinematics and, in fact, also show no signs of Na I or KI lines in their spectra. The objects are all relatively blue $(V-I)_{0} \leq 1.5$ and therefore represent strong candidate (metal-poor) M31 halo red giants in this field. This is an important result in itself as it represents the first detection of the metal-poor, stellar halo of M31 in the north-east quadrant.

\subsection{Intrinsic Velocity Dispersion and $M / L$ Ratio}

We calculate the velocity dispersion of And X by first accounting for the random and systematic velocity measurement errors as discussed in $\S 3.1$. The observed dispersion is the sum of this error and the true, intrinsic velocity dispersion of the galaxy. Using the maximumlikelihood method described by Walker et al. (2006), we derive $\sigma_{v}=3.9 \pm 1.2 \mathrm{~km} \mathrm{~s}^{-1}$ for the 22 And X member stars. The galaxy therefore ranks as the second coldest (kinematically) dSph in the Local Group. The current record belongs to the Milky Way satellite Leo IV, for which Simon \& Geha (2007) derive $\sigma_{v}=3.3 \pm 1.7 \mathrm{~km} \mathrm{~s}^{-1}$ based on 18 member stars. We note that the measured dispersion of And $\mathrm{X}$ is relatively insensitive to an (unknown) red giant binary fraction of $20-30 \%$. As Olszewski, Pryor, \& Armandroff (1996) have shown, unresolved binaries alone can inflate the measured velocity dispersion by as much $1.5 \mathrm{~km} \mathrm{~s}^{-1}$. Such an effect would reduce our measured velocity dispersion from 3.9 to $3.6 \mathrm{~km} \mathrm{~s}^{-1}$, a small change relative to the error bar in our measurement.

Such a low velocity dispersion for And $\mathrm{X}$ is almost a factor of two smaller than the "floor" suggested by Gilmore et al. (2007) at $\sim 7 \mathrm{~km} \mathrm{~s}^{-1}$, based on previously studied dSphs. In their study of newly discovered dSphs, Simon \& Geha (2007) found four Milky Way satellites with velocity dispersions significantly less than this floor, Leo IV, Coma Berenices, Canes Venatici II, and Hercules. However, all four of these systems are considered very low-luminosity galaxies, with integrated brightnesses of just $-5.9<M_{V}<-3.7$, and therefore are different from the existing sample of brighter galaxies. An intrinsic velocity dispersion of $\sigma_{v}=3.9 \pm 1.2 \mathrm{~km} \mathrm{~s}^{-1}$ for And X not only implies that such systems exist outside of the Milky Way's dSphs, but also presents the first evidence that such kinematically cold populations may exist in much brighter galaxies (And $\mathrm{X}$ has an observed luminosity of $\left.M_{V}=-8.1 \pm 0.5\right)$.

We can estimate the mass of And X using the method described by Illingworth (1976), $M=167^{*} \beta r_{c} \sigma^{2}$, where $\beta=8$ for dSphs and $\sigma$ is the central velocity dispersion. The mean radius of our 22 member stars is equal to the core radius, and therefore the central velocity dispersion that is appropriate for this equation may be somewhat higher than the estimate from our sample. Note that this method also explicitly assumes that the mass and light trace one another, which may not be correct (see below and $\S 5$ for more discussion on this and other possibly incorrect assumptions). The core radius of And $\mathrm{X}$ is $271 \mathrm{pc}$ (Zucker et al. 2007), and therefore we find $M=$ $5.4_{-2.8}^{+3.8} \times 10^{6} M_{\odot}$. The mass of the galaxy is reasonably similar to the group of four Milky Way dSphs with low intrinsic velocity dispersions discussed above, all of which have $M=1-7 \times 10^{6} M_{\odot}$ calculated in the same way. Of course, given that the integrated luminosity of And X is much larger than these systems, the galaxy's massto-light ratio is smaller by a factor of 4 (in the case of Leo IV) to 12 (in the case of Coma Berenices) than the Milky Way low-luminosity dSphs. Formally, we calculate the mass-to-light ratio of And X to be $M / L_{V}=37_{-19}^{+26}$ in solar units.

We can also compare And X's dark matter content to Milky Way satellites of comparable luminosity, calculated in a similar way. Both Draco and Ursa Minor are metal-poor and have $M_{V} \sim-8.5$, yet their mass-to-light ratios are $M / L_{V} \gtrsim 80$ (Mateo 1998; Odenkirchen et al. 2001; Lokas 2009). Given the small difference in luminosity, these galaxies therefore contain roughly four times as much mass as And X. Two of the classical Milky Way dSphs with similar mass-to-light ratios to And X are Carina and Sextans (both with $M / L_{V}=30-40$ ), however these systems are $\sim 3 \times$ brighter than And X. We discuss the possible implications of these results further in $\S 5$.

It is important to note the limitations of the method described above to calculate mass-to-light ratios, not just for And X but also for many of the other Local Group dSphs. A central velocity dispersion from a few dozen measurements alone offers degenerate constraints on the dark matter mass of a galaxy (e.g., Zentner \& Bullock 2003). We have assumed that the galaxy is spherical, in dynamical equilibrium, and has an isotropic velocity dispersion, all of which may not be true (Simon \& Geha 2007). As mentioned above, we have also assumed that mass traces light.

A more accurate measure of the amount, and nature, of dark matter in these systems requires a full velocity dispersion profile (e.g., Strigari et al. 2007; 2008). This modeling includes a maximum likelihood analysis and full marginalization over the dark matter halo profile shape and the stellar velocity anisotropy, and benefits from large samples of accurate radial velocity measurements (specifically out to large radii). Of course, a different set of assumptions related to the Jeans mass modeling affects these measurements. We discuss the prospects for achieving such data sets for the M31 system of dwarf satellites in $\S 5$. For our And X data set, detailed modeling along these lines will be presented in J. Wolf (2009, in preparation). Preliminary results sug- 

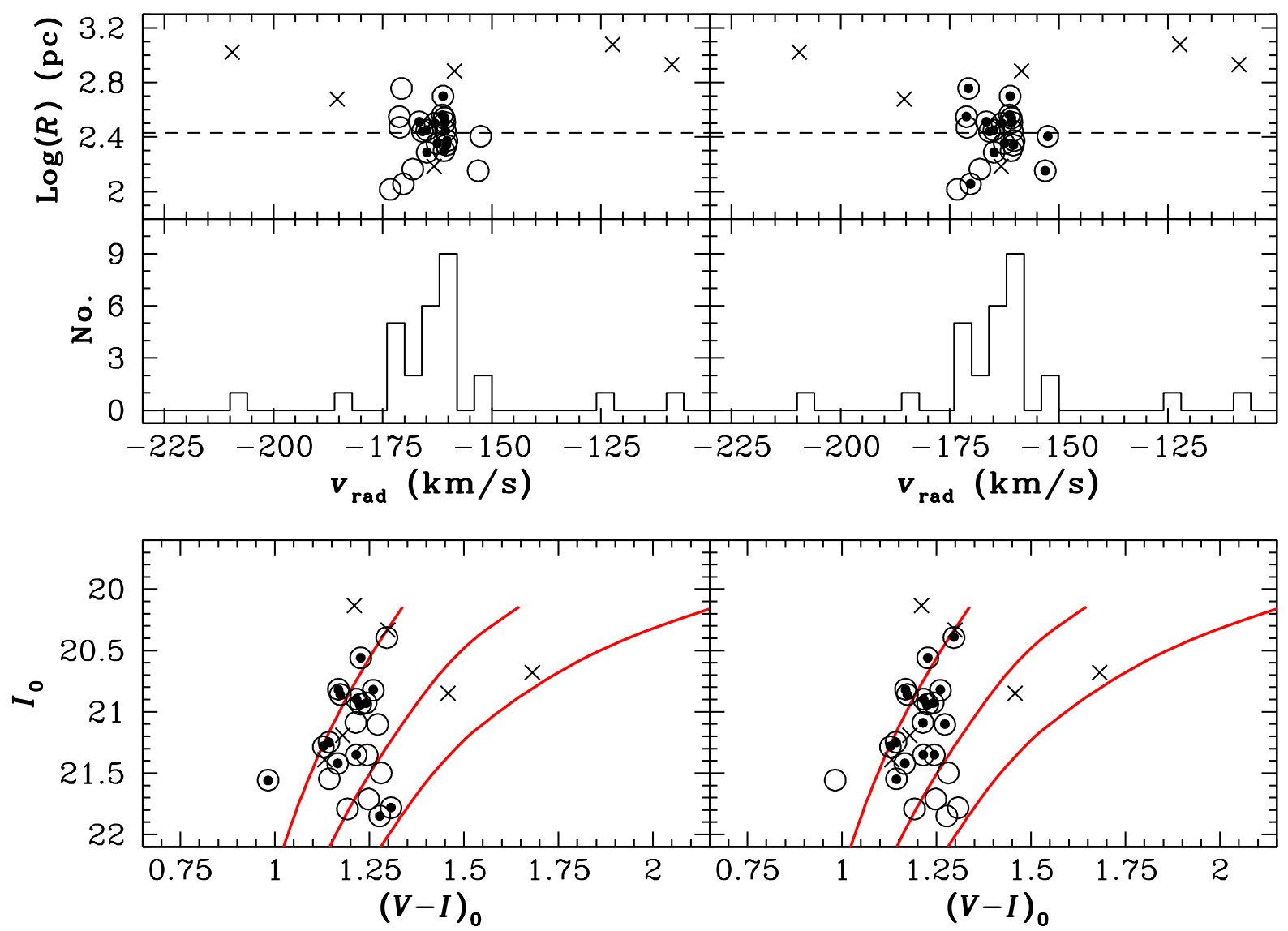

FIG. 4.- A diagnostic plot comparing specific characteristics of stars along the line of sight, including their radial distance from the center of And X vs the radial velocity (top panels, dashed line marks the core radius), a radial velocity histogram (middle panels), and the location of these stars on the CMD (bottom panels). The open circles denote our 22 member stars of And X, the open circles enclosing filled circles are the specific selections being analyzed (see below), and the crosses mark the six objects that are eliminated from the sample as discussed in $\S 3.2$. First, in the left panel, we isolate a core group of stars that have velocities in excellent agreement with the peak in the velocity histogram (formally within $3 \sigma$ of the measured And X mean radial velocity of $v_{\mathrm{rad}}=-163.8 \pm 1.2 \mathrm{~km} \mathrm{~s} \mathrm{~s}^{-1}$ ). (These dotted circles are difficult to discern on the top panel as they all reside in a narrow velocity range.) The distribution of these stars on the CMD in the bottom panel illustrates that the stars with blue and red colors at the faint end of our sample are in fact not outliers in the velocity diagnostic. In the right hand panels, the stars are selected to reside within the tight red giant branch sequence (excluding the fainter stars) and the resulting distribution in the radius vs velocity plane does in fact include some of the members in the wings of the histogram. These comparisons illustrate that the confirmed sample of stars is robust.

gest that the total mass (poorly constrained) out to the tidal radius in this galaxy is larger than the rough estimate given above. However, the mass at the half light radius, with much better constraints, is in fact systematically lower than the Milky Way counterparts of similar luminosity as reported above (modeled the same way).

\section{CHEMICAL ABUNDANCES}

\subsection{Photometric and Spectroscopic Metallicities}

The present day chemical abundances of dSphs are important tracers of their integrated star formation histories. The mean abundances, and abundance spreads, vary tremendously from one dwarf to another, which suggests that some galaxies were able to retain their enrichment products more efficiently than others. The CMD presented in Figure 3 shows that And $\mathrm{X}$ is a metal-poor system (the middle isochrone for 12 Gyr has $[\mathrm{Fe} / \mathrm{H}]=-1.31)$. We can measure the mean metallicity of the system by interpolating the positions of the stars on the CMD within a fine grid of stellar isochrones. Over the range $-2.31<[\mathrm{Fe} / \mathrm{H}]<-0.83$, the Vanden- berg, Bergbusch, \& Dowler (2006) models consist of a dozen such models. For the few stars just to the left of the most metal-poor isochrone, we obtain an $[\mathrm{Fe} / \mathrm{H}]$ value by slightly extrapolating the grid. The resulting metallicity distribution function is shown in Figure 5, assuming purely old ages (see below) and both $[\alpha / \mathrm{Fe}]=$ 0.0 isochrones (solid) and alpha-enhanced models with $[\alpha / \mathrm{Fe}]=+0.3$ (dashed). The median metallicity of the 22 confirmed members of And $\mathrm{X}$ is $[\mathrm{Fe} / \mathrm{H}]_{\mathrm{phot}}=-1.93$ \pm 0.11 for $[\alpha / \mathrm{Fe}]=0.0$ and $[\mathrm{Fe} / \mathrm{H}]_{\text {phot }}=-2.13 \pm 0.11$ for $[\alpha / \mathrm{Fe}]=+0.3$. The mean metallicities are consistent within the $1 \sigma$ uncertainties of these median values.

The absolute photometric uncertainty in color for the bulk of our stars with $I_{0} \lesssim 21.5$ is $\sim 0.05$ mags, which translates to a metallicity error of $\sigma[\mathrm{Fe} / \mathrm{H}] \sim 0.20$ at the bright, metal-poor end of the CMD. The CMD of And $\mathrm{X}$ members presented earlier in $\S 3.2 .1$ also illustrates that the uncertainty may be larger than this for the faintest stars in our member sample, with four of them falling significantly redder than the remaining sequence and one star lying much bluer than the most metal-poor 


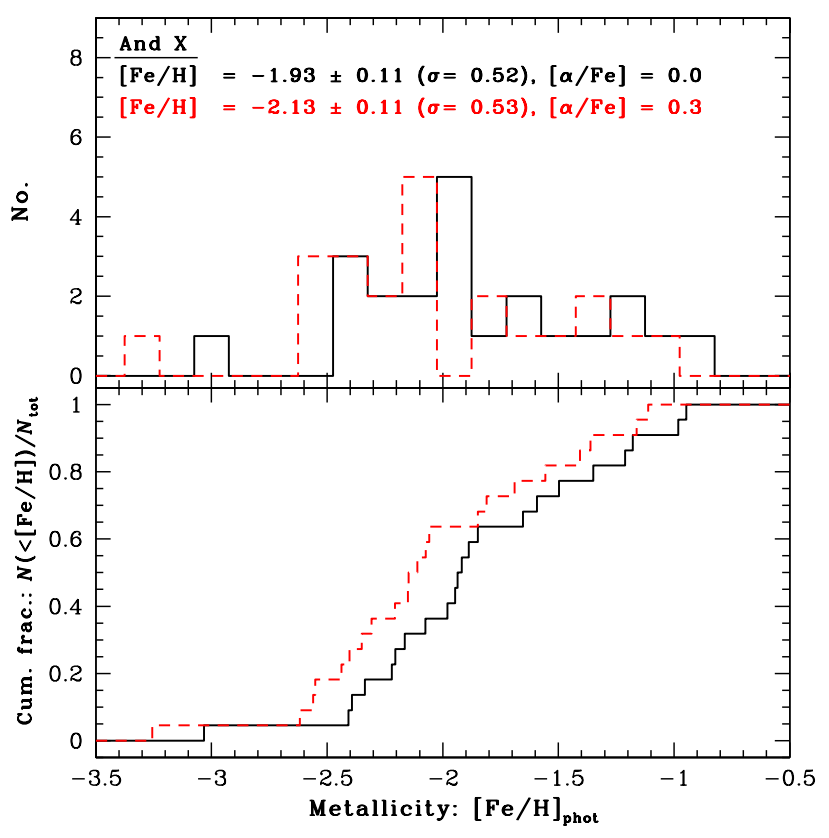

FIG. 5.- The metallicity distribution function is calculated by interpolating the photometry of confirmed And X stars within a grid of isochrones, assuming both $[\alpha / \mathrm{Fe}]=0.0$ (solid) and $[\alpha / \mathrm{Fe}]=$ +0.3 (dashed). The differential histogram is shown at the top and the cumulative distribution is shown in the bottom panel. And $\mathrm{X}$ is clearly a very metal-poor galaxy, with $[\mathrm{Fe} / \mathrm{H}]=-1.9$ to -2.1 , depending on the assumed $\alpha$-abundance. The intrinsic spread in chemical abundances is also large, $\sigma=0.48 \mathrm{dex}$. We compare these measurements to independently determined spectroscopic metallicities in Figure 6.

isochrone. If we eliminate these five stars from our metallicity analysis, the median and mean metallicity of And X remains essentially the same, changing by $<0.02 \mathrm{dex}$.

Our inferred metallicity of And X is based on photometric measurements of confirmed member red giant branch stars. This method assumes an input age and $[\alpha / \mathrm{Fe}]$. Unfortunately, there is currently no direct constraint on either of these parameters for And X. For the age, such a measurement would require deeper imaging to detect the morphology of the horizontal branch or mainsequence turnoff. We make the assumption that most of the stars in the galaxy are old based on both the fact that this is true for most other dSphs whose star formation histories have been measured (e.g., Mateo 1998; Harbeck et al. 2001), and because our deeper CMD does not show any evidence for a pronounced population of thermally pulsating asymptotic giant branch stars or a vertical red clump (see Nordic Optical Telescope data in Figure 3 of Zucker et al. 2007). Even if this assumption is wrong, our metallicity scale will not be drastically affected. For a shift in age from $12 \mathrm{Gyr}$ to $6 \mathrm{Gyr}$, the mean metallicity of a (metal-poor) star near the tip of the red giant branch would shift by only +0.25 dex. Similar to the uncertainty in age, the detailed chemical abundances of these stars are not known. For the Milky Way dSphs, $[\alpha / \mathrm{Fe}]$ can be measured by analyzing high resolution spectra of individual red giants. Studies of these stars in several Galactic dSphs (e.g., Draco, Sextans, and Ursa Minor) initially suggested that these objects are slightly enhanced in $\alpha$-elements, $0.02 \lesssim[\alpha / \mathrm{Fe}] \lesssim 0.13$ (Shetrone, Cote,\& Sargent 2001), however recent work
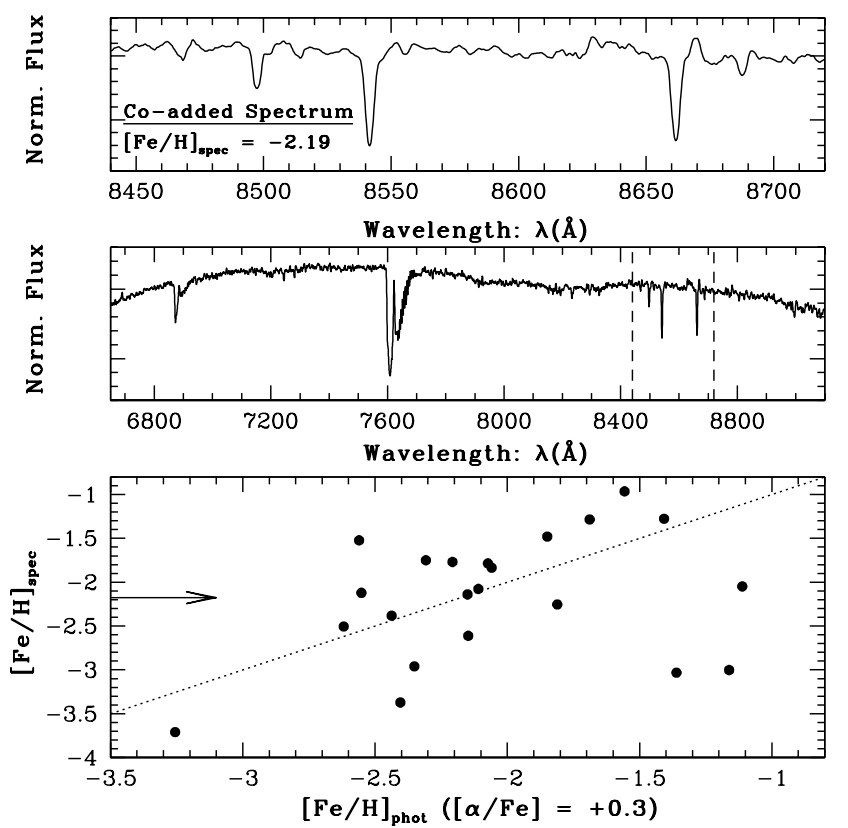

FIG. 6. - Bottom - The photometric metallicities calculated in $\S 4$ (for the $[\alpha / \mathrm{Fe}]=+0.3$ isochrones) are compared to independently measured spectroscopic estimates based on a calibration of the equivalent width of the Ca II triplet absorption lines. The straight dotted line represents the 1:1 relation and the arrow marks the mean spectroscopic abundance, $[\mathrm{Fe} / \mathrm{H}]_{\mathrm{spec}}=-2.18 \pm$ 0.15 . The middle panel exhibits the co-added spectrum of the 22 individual member red giants in And X. A zoomed view of this spectrum, over the region marked by the two vertical dashed lines is shown in the top panel. This high signal-to-noise spectrum yields a metallicity of $[\mathrm{Fe} / \mathrm{H}]_{\mathrm{spec}}=-2.19$, consistent with both the mean of the individual stars and also the photometric metallicity (see discussion in $\S 4$ ).

has found solar and/or slightly subsolar values (e.g., Cohen \& Huang 2009 and references therein). As Figure 5 demonstrates, such enhancement leads to a mean abundance that is more metal-poor than measured using our standard set of solar-scaled isochrones. Taken together, although it is clear that we currently have no direct constraints on either age or $\alpha$-enhancement in And X, we can qualitatively say that any reasonable offsets of these parameters from the values assumed would not cause large changes in our metallicity estimates.

An alternative method to determine the metallicity of red giant stars is to analyze their spectral features. Unfortunately, at the distance of And X the signal-to-noise of the individual spectra are too low to accurately characterize many absorption lines. The only spectral features that can be used to estimate $[\mathrm{Fe} / \mathrm{H}]$ from these data are the Ca II triplet at 8498,8542 , and $8662 \AA$ (see Rutledge, Hesser, \& Stetson 1997; Rutledge et al. 1997 for more information). Still, these lines reside in a region of the spectrum that contains many night sky lines and therefore the measured equivalent widths have large errors and the scatter in the spectroscopic metallicity measurements will be inflated (see e.g., Kalirai et al. 2006a). Although it is difficult to characterize the uncertainties, we nevertheless show the comparison of our measured $[\mathrm{Fe} / \mathrm{H}]_{\text {phot }}$ values to $[\mathrm{Fe} / \mathrm{H}]_{\text {spec }}$ measurements calculated in this way in Figure 6 (bottom). The empirical relation used in this calculation is derived using Galactic globular clusters and therefore the comparison has been made to 
the photometric metallicities assuming the $[\alpha / \mathrm{Fe}]=+0.3$ isochrones. We also point out that most globular clusters are more metal-rich than $[\mathrm{Fe} / \mathrm{H}] \sim-2.2$, and therefore this calibration is extrapolated for very metal-poor stars. Koch et al. (2008b) demonstrate that this leads to an underestimate of the true metallicity by several tenths of dex for very metal-poor stars. Further details on this empirical calibration can be found in Rutledge et al. ${ }^{16}$. The dotted line represents the 1:1 relation, and in general, the two independently determined metallicity values are found to agree well, although the scatter is large. The arrow marks the mean metallicity of the spectroscopic measurements, $[\mathrm{Fe} / \mathrm{H}]_{\text {spec }}=-2.18 \pm 0.15$, which is very similar to our photometric metallicity for $[\alpha / \mathrm{Fe}]=+0.3$ $\left([\mathrm{Fe} / \mathrm{H}]_{\text {phot }}=-2.13 \pm 0.11\right)$.

Figure 6 (bottom) can also be used to take a closer look at the five stars with possible poor photometry discussed above. Interestingly, the one very blue star in our CMD with $[\mathrm{Fe} / \mathrm{H}]_{\text {phot }}=-3.26$ for $[\alpha / \mathrm{Fe}]=+0.3$ is also found to be spectroscopically metal-poor. This star therefore represents a very interesting, and rare, metal-poor candidate in a dSph. Helmi et al. (2006) compare the metallicity distribution function of Galactic dSphs with the Galactic halo, and find a lack of $[\mathrm{Fe} / \mathrm{H}]<-3$ stars in the dSph population, suggesting that these satellites are different from the building blocks of the halo (but, also see Kirby et al. 2008). A higher signal-to-noise spectrum of this star can yield a more accurate spectroscopic metallicity. Contrary to what we find for this bluer star, the spectroscopic metallicities do not agree with the photometric estimates for three of the four faint stars that appear to be redder than the brighter, well defined red giant branch in Figure 3. These three stars are shown at the metal-rich end of the photometric scale on Figure 6 , and clearly fall well below the 1:1 relation. This comparison provides further evidence that the colors of these four stars are likely incorrect by at least $\sim 0.2$ magnitudes, and that the actual metallicities of the stars are in fact more metal-poor than we have determined from the isochrone analysis. Of course, if we just eliminate these three stars from the analysis, keeping the one blue star in our sample, the mean metallicity of And $\mathrm{X}$ becomes more metal-poor by $\sim 0.1$ dex compared to the values given above.

In the top two panels of Figure 6 we present a high signal-to-noise co-added spectrum of the 22 individual spectra for confirmed And X members. This was constructed by weighting the individual spectra by the inverse variance squared to minimize the contribution from features affected by bad pixels or residual night sky lines. The middle panel shows the full spectrum and the top panel shows the spectrum in the wavelength range of the Ca II triplet (i.e., the dashed lines in the middle panel). The Ca II triplet lines in this co-added spectrum are well defined and therefore the equivalent widths can be measured to greater precision than in the individual spectra. Using the same method as above on this single co-added spectrum, we find a value for the spectroscopic metallicity of $[\mathrm{Fe} / \mathrm{H}]_{\mathrm{spec}}=-2.19$, essentially identical to the mean of the individual measurements. Given the method used to co-add the individual spectra, we do not have

\footnotetext{
16 We have assumed the luminosity of And X's horizontal branch to be $V_{\mathrm{HB}}=25.0$.
}

any information on the spread in abundances specifically from this measurement.

To summarize, we have used two independent diagnostics to determine the chemical abundance of And X. Each of these two methods has different disadvantages. For example, the photometric measurement is sensitive to the quality of the photometry and the spectroscopic metallicity is affected by the low signal-to-noise of our spectra. Nevertheless, the results from the two methods are found to be in good agreement. As we do not know whether stars in this galaxy are $\alpha$-enhanced, we adopt the photometric metallicity for $[\alpha / \mathrm{Fe}]=0.0$ as our best value, $[\mathrm{Fe} / \mathrm{H}]_{\text {phot }}=-1.93 \pm 0.11$, and note that the metallicity would be more metal-poor by $\sim 0.1$ dex if the galaxy is significantly enhanced in $\alpha$-elements (i.e., $[\alpha / \mathrm{Fe}]=+0.3)$.

Based on these calculations, And $\mathrm{X}$ is among the most metal-poor nearby dSph galaxies with $M_{V} \lesssim-8$ (e.g., Table 1 in Grebel, Gallagher, \& Harbeck 2003). In terms of the well-known correlation between the luminosity of a dwarf galaxy and its metallicity over this range (Aaronson 1986; Mateo 1998), And X sits near the previously established relation for dSphs, anchoring the faint, metalpoor end. A strong deviation from this relation (e.g., a dwarf that is too faint for its metallicity) may suggest that the galaxy lost some of its stellar component through tidal disruption. Our metallicity analysis does not provide any strong evidence that And X's present day population has been affected in this way. Of course, Simon \& Geha (2007) have shown that the metallicities of the newly discovered SDSS low-luminosity galaxies with $-8 \lesssim M_{V} \lesssim-3.5$ may be even more metal-poor than $[\mathrm{Fe} / \widetilde{\mathrm{H}}] \sim-\widetilde{2}$ (see also Kirby et al. 2008).

Derived parameters for each of the 22 confirmed red giants in And X are presented in Table 2.

\subsection{Metallicity Spread and Radial Metallicity Gradient}

The total measured abundance dispersion for And $\mathrm{X}$ is relatively large, $\sigma\left([\mathrm{Fe} / \mathrm{H}]_{\text {phot }}\right)=0.52$. This dispersion represents a combination of the true intrinsic dispersion of the galaxy and the scatter introduced by photometric errors. If we subtract off the error term in quadrature $(\sim 0.2 \mathrm{dex})$, the resulting intrinsic abundance dispersion is calculated to be $\sigma\left([\mathrm{Fe} / \mathrm{H}]_{\text {phot }}\right)=0.48$. This is not unlike typical values found for Milky Way dSphs (Mateo 1998) and suggests that And $\mathrm{X}$ was able to retain much of its enrichment products. A full interpretation of this will of course benefit greatly from knowledge of the star formation history of the galaxy, as measured from deeper photometric observations. For example, if the galaxy shows evidence for an extended epoch of star formation as seen in Fornax and Leo I, then a small fraction of the spread in color on the upper red giant branch can be explained by age differences. We also note that with a sample of just 22 stars, our results are mildly sensitive to any extreme outliers in the metallicity distribution. If we remove the three objects with discrepant photometric vs spectroscopic metallicities discussed above, the dispersion decreases to $\sigma\left([\mathrm{Fe} / \mathrm{H}]_{\text {phot }}\right)=0.38$.

In Figure 7 we illustrate the photometric (top panel) and spectroscopic (bottom panel) metallicities of stars as a function of their distance from the center of And X. The core radius of the galaxy, $r_{\mathrm{c}}=271 \mathrm{pc}$ is marked with a dashed line. There is clear evidence of a radial metallicity 


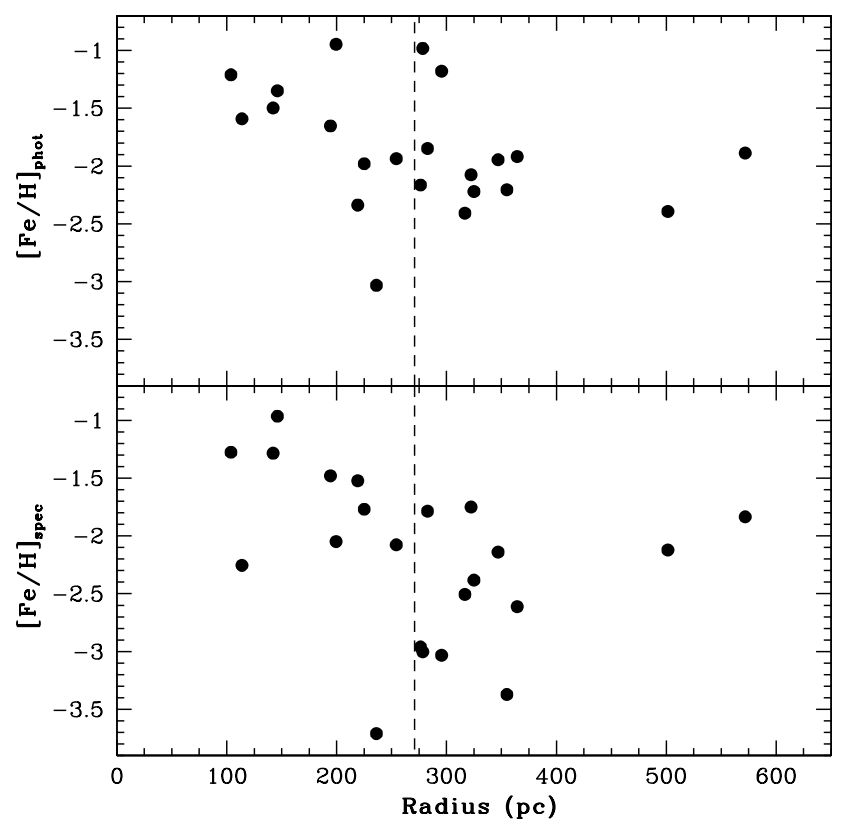

FIG. 7.- The photometric (top) and spectroscopic (bottom) metallicities indicate the presence of a radial abundance gradient in And X. The inner regions of the $\mathrm{dSph}$ are clearly more metal-rich than stars near the core radius $\left(r_{\mathrm{c}}=271 \mathrm{pc}\right.$, dashed line).

gradient in And X, with more metal-rich stars being centrally located. These innermost stars have $[\mathrm{Fe} / \mathrm{H}]_{\text {phot }} \sim$ -1.4 which gradually decreases to $[\mathrm{Fe} / \mathrm{H}]_{\text {phot }} \lesssim-2.0$ near, and beyond, the core radius. We also note that two of the faintest four stars in our data set with redder $V-I$ colors are located at $r \sim 300 \mathrm{pc}$, and therefore these stars do not drive the observed gradient.

In order to distinguish between different formation scenarios of this galaxy, the abundance gradient should be verified using deeper photometry to detect a morphological change in the horizontal branch of the galaxy as a function of radius. The result could also suggest that an age gradient is the driver of the observed metallicity gradient, which can be tested with photometric studies of the main-sequence turnoff of And X. Harbeck et al. (2001) note that of the other M31 dSphs that have been studied photometrically down to the horizontal branch, only And I and VI show an obvious population gradient.

\section{DISCUSSION AND SUMMARY}

And $\mathrm{X}$ has an integrated magnitude $\left(M_{V}=-8.1 \pm\right.$ 0.5 ) that bridges the luminosity of the Local Group dwarf galaxies known prior to SDSS $\left(-15.5<M_{V}<-8.5\right)$, and those since discovered $\left(-8<M_{V}<-3.5\right)$. Over this factor of nearly $10^{5}$ in luminosity (more than 12 magnitudes), these galaxies show a remarkable trend with more more luminous dwarfs also being more metal-rich (see, e.g., Figure 11 in Simon \& Geha 2007). We have shown - using both photometric and spectroscopic diagnostics - that the stellar populations in And X are metal-poor, with $[\mathrm{Fe} / \mathrm{H}]=-1.9$ to -2.1 , depending on the method adopted and/or assumption of $[\alpha / \mathrm{Fe}]$. The galaxy therefore anchors the low luminosity end of the classical satellites, and has a mean metallicity consistent with the established trend. When we also include the new SDSS galaxies, And X is well placed within the overall sequence of data points and does not appear to be underluminous relative to its metallicity (which could have suggested evidence of tidal stripping).

Our kinematic analysis of And X shows that the galaxy is moving with a heliocentric radial velocity of $v_{\mathrm{rad}}=$ $-163.8 \pm 1.2 \mathrm{~km} \mathrm{~s}^{-1}$, and has a very low intrinsic velocity dispersion, $\sigma_{v}=3.9 \pm 1.2 \mathrm{~km} \mathrm{~s}^{-1}$. Compared to similar luminosity Milky Way dSphs such as Carina, Draco, Ursa Minor, and Canes Venatici I, And X's intrinsic velocity dispersion is a factor of $1.7-2.4$ smaller. Although its size is larger than Carina, Draco, and Ursa Minor by a similar factor (see below), the ratio of $r_{c} \sigma^{2}$ comes out lower. We use this to estimate the mass of And X (assuming mass follows light) and find $M=5.4_{-2.8}^{+3.8} \times 10^{6} M_{\odot}$, implying a mass-to-light ratio of $M / L_{V}=37_{-19}^{+26}$. The dark matter mass of And $\mathrm{X}$ is therefore approximately a factor of four lower than Milky Way dSphs with comparable, but slightly higher, luminosity (see $\S 3.3$ ).

It is difficult to draw strong conclusions on the possible implications of the mass difference between And X and similar Milky Way satellites. The differences may simply reflect variations of in situ processes, in which case And X may be an oddball with respect to the small comparison sample of galaxies at similar luminosities. Alternatively, if And X is a typical representative of the M31 dSph sample, the observed differences may have been possibly shaped by intrinsic differences in the overall properties of the Milky Way and M31 halos. For example, the past interaction history of these dSphs with their hosts are likely different in the two systems as M31 appears to have a more violent accretion history (Hammer et al. 2007). Whether or not this could lead to preferentially more stripping of the dark matter halos of the surviving satellites such as And X is not certain, especially within the visible radius of the galaxy (e.g., stars should also be stripped then). Hints of overall global differences between M31 and the Milky Way have already been characterized, on several fronts. For example, M31 contains many more globular clusters than the Milky Way (e.g., Barmby \& Huchra 2001), M31's stellar density at $20 \mathrm{kpc}$ along the minor axis is $10 \times$ higher than the Milky Way (Reitzel, Guhathakurta, \& Gould 1998), and M31's halo contains a vast amount of substructure extending out to very large radii (Ibata et al. 2007). Further differences are seen in the overall age, metallicity, and surface brightness distributions, as characterized in our overall SPLASH Survey. For example, M31's halo contains an appreciable fraction of stars with ages less than $10 \mathrm{Gyr}$ out to $20 \mathrm{kpc}$ along the minor axis (Brown et al. 2007), and the radius at which the metal-poor halo dominates the metal-rich inner spheroid is much further out in M31 than in the Milky Way (Guhathakurta et al. 2005; Kalirai et al. 2006a).

Interestingly, there is another piece of evidence suggesting systematic differences between the dSph populations of the Milky Way and M31. As we mentioned in $\S 1$, McConnachie \& Irwin (2006) analyzed photometric data of a half dozen M31 dSphs and found that at a fixed luminosity, these satellites are larger by a factor of two and have fainter central surface brightnesses than Milky Way satellites. And $\mathrm{X}$ is not included in that sample, however it does appear to follow the same trend. The half light radius is $330 \mathrm{pc}$ and tidal radius is $1500 \mathrm{pc}$, 
TABLE 2

\begin{tabular}{|c|c|c|c|c|c|c|c|c|c|}
\hline$\overline{\text { Mask }}$ & $\alpha_{J 2000}$ & $\overline{\delta_{J 2000}}$ & $\overline{I_{0}}$ & $\overline{(V-I)_{0}}$ & $r(\mathrm{pc})$ & $v_{\text {rad }}\left(\mathrm{km} \mathrm{s}^{-1}\right)$ & $\begin{array}{c}{[\mathrm{Fe} / \mathrm{H}]_{\text {phot }}} \\
([\alpha / \mathrm{Fe}]=0.0)\end{array}$ & $\begin{array}{c}{[\mathrm{Fe} / \mathrm{H}]_{\text {phot }}} \\
([\alpha / \mathrm{Fe}]=0.3)\end{array}$ & $\overline{[\mathrm{Fe} / \mathrm{H}]_{\mathrm{spec}}}$ \\
\hline d10_1 & $01: 06: 30.83$ & $+44: 47: 50.2$ & 20.39 & 1.30 & 355 & $-171.2 \pm 3.1$ & -2.20 & -2.40 & -3.37 \\
\hline d10_1 & $01: 06: 28.43$ & $+44: 47: 11.9$ & 20.56 & 1.23 & 501 & $-161.2 \pm 3.2$ & -2.39 & -2.55 & -2.12 \\
\hline d10_1 & 01:06:29.79 & $+44: 48: 25.1$ & 20.82 & 1.26 & 347 & $-160.9 \pm 4.4$ & -1.95 & -2.15 & -2.14 \\
\hline d10_2 & 01:06:46.08 & $+44: 47: 47.0$ & 20.82 & 1.17 & 317 & $-163.0 \pm 4.2$ & -2.41 & -2.62 & -2.51 \\
\hline d10_2 & $01: 06: 38.74$ & $+44: 47: 40.3$ & 20.86 & 1.17 & 219 & $-160.4 \pm 2.7$ & -2.34 & -2.56 & -1.52 \\
\hline d10_2 & $01: 06: 34.60$ & $+44: 47: 23.4$ & 20.90 & 1.22 & 322 & $-160.8 \pm 4.2$ & -2.08 & -2.31 & -1.75 \\
\hline d10_2 & $01: 06: 32.98$ & $+44: 48: 43.6$ & 20.94 & 1.23 & 225 & $-162.5 \pm 3.4$ & -1.98 & -2.21 & -1.77 \\
\hline d10_2 & $01: 06: 34.21$ & $+44: 47: 51.9$ & 21.09 & 1.21 & 254 & $-152.5 \pm 3.1$ & -1.94 & -2.11 & -2.08 \\
\hline d10_2 & $01: 06: 36.48$ & $+44: 48: 27.8$ & 21.10 & 1.27 & 114 & $-170.3 \pm 3.1$ & -1.59 & -1.81 & -2.25 \\
\hline d10_2 & 01:06:45.98 & $+44: 48: 07.2$ & 21.25 & 1.14 & 276 & $-165.9 \pm 4.3$ & -2.16 & -2.35 & -2.96 \\
\hline d10_2 & $01: 06: 34.27$ & $+44: 47: 24.7$ & 21.28 & 1.13 & 325 & $-166.6 \pm 3.5$ & -2.22 & -2.44 & -2.38 \\
\hline d10_1 & 01:06:35.21 & $+44: 48: 06.3$ & 21.35 & 1.22 & 194 & $-164.8 \pm 2.7$ & -1.65 & -1.85 & -1.48 \\
\hline d10_2 & $01: 06: 35.70$ & $+44: 48: 25.6$ & 21.35 & 1.24 & 142 & $-153.1 \pm 4.9$ & -1.50 & -1.69 & -1.28 \\
\hline d10_2 & $01: 06: 36.37$ & $+44: 48: 39.5$ & 21.50 & 1.28 & 104 & $-173.3 \pm 4.3$ & -1.21 & -1.41 & -1.28 \\
\hline d10_1 & $01: 06: 24.40$ & $+44: 47: 46.0$ & 21.55 & 1.14 & 572 & $-170.7 \pm 6.7$ & -1.89 & -2.06 & -1.84 \\
\hline d10_1 & 01:06:32.70 & $+44: 48: 51.0$ & 21.56 & 0.98 & 236 & $-160.3 \pm 10.1$ & -3.03 & -3.26 & -3.71 \\
\hline d10_2 & 01:06:44.32 & $+44: 47: 36.9$ & 21.71 & 1.29 & 296 & $-171.1 \pm 5.1$ & -1.18 & -1.36 & -3.03 \\
\hline d10_2 & 01:06:33.85 & $+44: 48: 30.4$ & 21.78 & 1.31 & 200 & $-161.0 \pm 9.4$ & -0.95 & -1.11 & -2.05 \\
\hline d10_1 & $01: 06: 35.49$ & $+44: 48: 27.6$ & 21.79 & 1.19 & 146 & $-168.1 \pm 12.2$ & -1.35 & -1.56 & -0.96 \\
\hline d10_1 & 01:06:32.69 & $+44: 48: 01.0$ & 21.85 & 1.28 & 278 & $-160.6 \pm 5.8$ & -0.98 & -1.16 & -3.00 \\
\hline
\end{tabular}

both much larger than the corresponding sizes of similar luminosity Milky Way satellites. Yet, as discussed above, the very low intrinsic velocity dispersion of And $\mathrm{X}$ more than compensates for its larger size in yielding a total mass that is lower than these Milky Way satellites.

And X's mass measurement depends critically on our assumptions related to how the dark matter mass links to the stellar distribution; namely, we have adopted the formalism that the mass traces light. A different interpretation of the kinematics would result from an overall different spatial segregation. For example, Penarrubia, McConnachie, \& Navarro (2007) compute models in which the kinematics are dictated by the ratio of the King core radius (stellar) to the NFW scale radius (dark matter), and find that a more concentrated distribution exhibits a lower velocity dispersion. With this assumption, less dark matter mass is needed in the larger galaxies to explain the same observed velocity dispersion. Penarrubia et al. go on to predict that, assuming M31 and Milky Way dSphs have the same overall dark matter mass, the velocity dispersions of the M31 satellites should be larger than the Milky Way satellites. Based on our sample of a single M31 dSph, our results do not agree with this prediction.

There are currently 17 known M31 dSphs, And I III, V - VII, IX - XVII, and XIX - XX, the last ten of which have been discovered in the past three years (Zucker et al. 2004b, 2007; Martin et al. 2006; Majewski et al. 2007; Ibata et al. 2007; Irwin et al. 2008; McConnachie et al. 2008). (The three systems not included, And IV, VIII and XVIII, are background or ambiguous objects originally identified as M31 dSphs.) Radial velocities of at least a few individual red giants have been measured in the first six of these galaxies (Cote et al. 1999; Guhathakurta, Reitzel, \& Grebel 2000; Evans et al. 2000). Unfortunately, with the exception of stars in And II and And VII, which were targeted with HIRES on Keck, the data were collected at both low resolution and low signal-to-noise, resulting in large velocity uncertainties, and therefore the intrinsic velocity dispersion of the satellites were not resolved. For the two galaxies observed with Keck/HIRES, the central velocity dispersion was estimated to be $\sim 9 \mathrm{~km} \mathrm{~s}^{-1}$ in both galaxies, based on seven stars in And II (Cote et al. 1999) and 18 stars in And VII (Guhathakurta, Reitzel, \& Grebel 2000; Kalirai et al. 2009). For the newly discovered SDSS satellite And IX (Zucker et al. 2004b), Chapman et al. (2005) present a Keck/DEIMOS study establishing just five member stars within the inner $1.4^{\prime}$ of the galaxy and possibly another six or seven members at larger radius. As the authors note, their results are very sensitive to the adopted membership criteria. The inclusion of just one star in the outer bin inflates the velocity dispersion by a factor of almost two, and therefore we can not reliably use this measurement until further data are obtained. Majewski et al. (2007) also present radial velocities for stars in the newly discovered galaxy And XIV, which we will analyze in Kalirai et al. (2009).

Clearly, all of these M31 dSphs need to be re-targeted to establish accurate kinematics based on larger numbers of stars. In addition, the newly detected objects that have not yet been looked at need to be observed. Our team has now begun a systematic survey to compile this data set, the first results from which will include an analysis of $50-100$ accurate radial velocities in each of And I, II, and III. We will also combine the results from the study of these three galaxies with a more detailed analysis of the results presented in Guhathakurta, Reitzel, \& Grebel (2000) for And VII, Majewski et al. (2007) for And XIV, and of course also include the present work on And X. As discussed earlier, future modeling of these dSphs will also incorporate advanced techniques such as those presented by Strigari et al. (2008) for Milky Way galaxies, allowing mass profiles to be constructed for each satellite. In this way, the dark matter masses of dSph members of the Milky Way and M31 can be compared out 
to the same physical radii. With a sample of six galaxies, these comparisons can also be made over a large range in luminosity and galactocentric radius, thereby providing more leverage on understanding the sources of any observed differences.

A detailed understanding of the dark matter mass profiles of the M31 dSphs will require significantly larger data sets involving accurate radial velocities of several hundred stars in each galaxy. Such data can provide information on how luminosity maps to dark matter subhalo mass and also shed light on the validity of alternative dark matter candidates such as "warm dark matter". These types of detailed studies are just now underway for several of the Milky Way dSphs (e.g., Strigari et al. 2006) and will soon be undertaken in the nearest, most luminous dSphs of M31. To this effort, we have now obtained the first wave of the data required to make these measurements; an analysis of $\sim 500$ individual radial velocities in the luminous dSph And II is underway.

We gratefully acknowledge E. Kirby for help with calculating photometric and spectroscopic metallicities, and M. Schirmer and T. Pursimo for assistance in reducing the William Herschel and Nordic Optical Telescope data. We wish to also thank J. Wolf for very helpful extensive discussions on the mass modeling of these kinematic data. Finally, we wish to thank an anonymous referee for many suggestions that have improved this paper.

JSK's research is supported in part by a grant from the STScI Director's Discretionary Research Fund, and was supported by NASA through Hubble Fellowship grant HF-01185.01-A, awarded by the Space Telescope Science
Institute, which is operated by the Association of Universities for Research in Astronomy, Incorporated, under NASA contract NAS5-26555. This project was also supported by NASA/STScI grant GO-10265.02 (JSK and PG). DBZ acknowledges support from a National Science Foundation International Postdoctoral Fellowship.

Funding for the SDSS and SDSS-II has been provided by the Alfred P. Sloan Foundation, the Participating Institutions, the National Science Foundation, the U.S. Department of Energy, the National Aeronautics and Space Administration, the Japanese Monbukagakusho, the Max Planck Society, and the Higher Education Funding Council for England. The SDSS Web Site is http://www.sdss.org/.

The SDSS is managed by the Astrophysical Research Consortium for the Participating Institutions. The Participating Institutions are the American Museum of Natural History, Astrophysical Institute Potsdam, University of Basel, University of Cambridge, Case Western Reserve University, University of Chicago, Drexel University, Fermilab, the Institute for Advanced Study, the Japan Participation Group, Johns Hopkins University, the Joint Institute for Nuclear Astrophysics, the Kavli Institute for Particle Astrophysics and Cosmology, the Korean Scientist Group, the Chinese Academy of Sciences (LAMOST), Los Alamos National L aboratory, the Max-Planck-Institute for Astronomy (MPIA), the MaxPlanck-Institute for Astrophysics (MPA), New Mexico State University, Ohio State University, University of Pittsburgh, University of Portsmouth, Princeton University, the United States Naval Observatory, and the University of Washington.

\section{REFERENCES}

Aaronson, M. 1986, In Star Forming Dwarf Galaxies and Related Objects, ed. D Kunth, TX Thuan, JTT Van, 125. Paris: Ed. Front.

Barmby, P., \& Huchra, J. P. 2001, AJ, 122, 2458

Belokurov, V., et al. 2006, ApJ, 647, L111

Belokurov, V., et al. 2007, ApJ, 654, 897

Brown, T., et al. 2007, ApJ, 658, L95

Chapman, S. C., Ibata, R. A., Lewis, G. F., Ferguson, A. M. N., Irwin, M., McConnachie, A. W., \& Tanvir, N. 2005, ApJ, 632, L87

Cohen, J. G., \& Huang, W. 2009, ApJ, 701, 1053

Cote, P., Mateo, M., Olszewski, E. W., \& Cook, K. H. 1999, ApJ, 526,147

Evans, N. W., Wilkinson, M. I., Guhathakurta, P., Grebel, E. K., \& Vogt, S. S. 2000, ApJ, 540, L9

Faber, S. M., et al. 2007, ApJ, 665, 265

Geha, M., Willman, B., Simon, J. D., Strigari, L., Kirby, E. N., Law, D. R., \& Strader, J. 2009, ApJ, 692, 1464

Gilbert, K. M., Guhathakurta, P., Kalirai, J. S., Rich, R. M., Majewski, S. R., Ostheimer, J. C., Reitzel, D. B., Cenarro, A. J., Cooper, M. C., Luine, C., \& Patterson, R. J. 2006, ApJ, 652, 1188

Gilmore, G., Wilkinson, M. I., Wyse, R. F. G., Kleyna, J. T., Koch, A., Evans, N. W., \& Grebel, E. K. 2007, ApJ, 663, 948

Grebel, E. K., Gallagher, J. S., III, Harbeck, D. 2003, AJ, 125, 1926

Guhathakurta, P., Reitzel, D. B., \& Grebel, E. K. 2000, Proc. SPIE, 4005, 168

Guhathakurta, P., Ostheimer, J. C., Gilbert, K. M., Rich, R. M., Majewski, S. R., Kalirai, J. S., Reitzel, D. B., \& Patterson, R. J. 2005, astro-ph/0502366

Guhathakurta, P., Rich, R. M., Reitzel, D. B., Cooper, M. C., Gilbert, K. M., Majewski, S. R., Ostheimer, J. C., Geha, M. C., Johnston, K. V., \& Patterson, R. J. 2006, AJ, 131, 2497

Hammer, F., Puech, M., Chemin, L., Flores, H., \& Lehnert, M. 2007, ApJ, 662, 322

Harbeck, D. et al. 2001, AJ, 122, 3092

Helmi, A., et al. 2006, ApJ, 651, L121
Horne, K. 1986, PASP, 98, 609

Ibata, R., Martin, N. F., Irwin, M., Chapman, S., Ferguson, A. M. N., Lewis, G. F., \& McConnachie, A. W. 2007, ApJ, 671, 1591

Illingworth, G. 1976, ApJ, 204, 73

Irwin, M. J., Ferguson, A. M. N., Huxor, A. P., Tanvir, N. R., Ibata, R. A., \& Lewis, G. F. 2008, ApJ, 676, L17

Kalirai, J. S., Gilbert, K. M., Guhathakurta, P., Majewski, S. R., Ostheimer, J. C., Rich, R. M., Cooper, M. C., Reitzel, D. B., \& Patterson, R. J. 2006a, ApJ, 648, 389

Kalirai, J. S., Guhathakurta, P., Gilbert, K. M., Reitzel, D. B., Rich, R. M., Majewski, S. R., \& Cooper, M. C. 2006b, ApJ, 641, 268

Kalirai, J. S., et al. 2009, in preparation

Keller, S. C., et al. 2007, PASA, 24, 1

Kirby, E., Simon, J., Geha, M., Guhathakurta, P., \& Frebel, A., 2008, ApJ, 685, 43

Kleyna, J. T., Wilkinson, M. I., Evans, N. W., \& Gilmore, G. 2005, ApJ, 630, L141

Klypin, A., Kravtsov, A. V., Valenzuela, O., \& Prada, F. 1999, ApJ, 522, 82

Koch, A., McWilliam, A., Grebel, E. K., Zucker, D. B., \& Belokurov, V. 2008a, ApJ, 688, L13

Koch, A., Grebel, E. K., Gilmore, G. F, Wyse, R. F. G., Kleyna, J. T., Harbeck, D. R., Wilkinson, M. I., \& Wyn, E. N. 2008b, AJ, 135,1580

Lokas, E. L. 2009, MNRAS, 394, L102

Majewski, S. R. et al. 2007, ApJ, 670, L9

Martin, N. F., Ibata, R. A., Irwin, M. J., Chapman, S., Lewis, G. F., Ferguson, A. M. N., Tanvir, N., \& McConnachie, A. W. 2006, MNRAS, 371, 1983

Mateo, M. 1998, ARA\&A, 36, 435

McConnachie, A. W., \& Irwin, M. J. 2006, MNRAS, 365, 1263

McConnachie, A. W., et al. 2008, ApJ, 688, 1009

Moore, B., Ghigna, S., Governato, F., Lake, G., Quinn, T., Stadel, J., \& Tossi, P. 1999, ApJ, 524, L19 
Norris, J. E., Gilmore, G., Wyse, R. F. G., Wilkinson, M. I., Belokurov, V., Evans, N. W., \& Zucker, D. B. 2008, ApJ, 689, L113

Odenkirchen, M. et al. 2001, AJ, 122, 2538

Olszewski, E. W., Pryor, C., \& Armandroff, T. E. 1996, AJ, 111, 750

Penarrubia, J., McConnachie, A., \& Navarro, J. F. 2007, ApJin press, astro-ph/0701780

Reitzel, D. B., Guhathakurta, P., \& Gould, A. 1998, AJ, 116, 707

Rutledge, G. A., Hesser, J. E., \& Stetson, P. B. 1997, PASP, 109, 907

Rutledge, G. A., Hesser, J. E., Stetson, P. B., Mateo, M., Simard, L., Bolte, M., Friel, E. D., \& Copin, Y. 1997, PASP, 109, 883

Shetrone, M. D., Cote, P., \& Sargent, W. L. W. 2001, ApJ, 548, 592

Simon, J., \& Geha, M. 2007, ApJ, 670, 313

Sohn, S. T., et al. 2007, ApJ, 663, 960

Somerville, R. S. 2002, ApJ, 572, L23

Strigari, L. E., Bullock, J. S., Kaplinghat, M., Kravtsov, A. V., Gnedin, O. Y., Abazajian, K., \& Klypin, A. A. 2006, ApJ, 652, 306
Strigari, L. E., Bullock, J. S., Kaplinghat, M., Diemand, J., Kuhlen, M., \& Madau, P. 2007, ApJ, 669, 676

Strigari, L. E., Bullock, J. S., Kaplinghat, M., Simon, J. D., Geha, M., Willman, B., \& Walker, M. G. 2008, Nature, 454, 1096

Tollerud, E. J., Bullock, J. S., Strigari, L. E., \& Willman, B. 2008, ApJ, 688, 277

VandenBerg, D. A., Bergbusch, P. A., \& Dowler, P. D. 2006, ApJS, 162,375

Walker, M G., Mateo, M., Olszewski, E. W., Bernstein, R., Wang, X., \& Woodroofe, M. 2006, AJ, 131, 2114

Walsh, S. M., Jerjen, H., \& Willman, B. 2007, ApJ, 662, L83

Willman, B., et al. 2005, ApJ, 626, L85

York, D.G., et al. 2000, AJ, 120, 1579

Zentner, A. R., \& Bullock, J. S. 2003, ApJ, 598, 49

Zucker, D. B. et al. 2004a, ApJ, 612, L117

Zucker, D. B. et al. 2004b, ApJ, 612, L121

Zucker, D. B. et al. 2006a, ApJ, 643, L103

Zucker, D. B. et al. 2006b, ApJ, 650, L41

Zucker, D. B. et al. 2007, ApJ, 659, L21 